\title{
Perfil epidemiológico de las enfermedades profesionales en Alicante, 2002-2005
}

\section{Epidemiological profile of occupational diseases in Alicante, 2002-2005}

\section{Senén Berenguer-Ortuño}

Unidad de Cuidados Críticos. Hospital Vega Baja.

Orihuela-Alicante. España

\section{Andrés A. Agudelo-Suárez}

Área de Medicina Preventiva y Salud Pública. Universidad de Alicante. Alicante. España

Facultad de Odontología. Universidad de Antioquia. Medellin. Colombia

\section{José María Roél-Valdés}

Centro Territorial INVASSAT Alicante. Conselleria de Economía,

Hacienda y Empleo. Generalitat Valenciana. España

\section{Elena Ronda-Pérez}

Área de Medicina Preventiva y Salud Pública. Universidad de Alicante. Alicante. España

\section{Correspondencia:}

Senén Berenguer-Ortuño

Hospital Vega Baja. Orihuela- Alicante.

Ctra Orihuela- Almoradí s/n

San Bartolomé- Orihuela. Alicante. España

Telf.: 34965877500 Ext. 52732

berenguer_sen@gva.es

Resumen

Objetivo: Describir el patrón epidemiológico de las enfermedades profesionales (EEPP) en la provincia de Alicante entre los años 2002-2005.

Métodos: Estudio transversal. La fuente de información procede de los partes del registro de EEPP notificadas en Alicante. Las enfermedades incluidas han sido los trastornos musculoesqueléticos (TME) del miembro superior, dermatitis y asma. Como factores laborales y personales se han considerado la edad, el sexo, el puesto de trabajo, el tamaño de la empresa, y la antigüedad laboral. Se han estimado las tasas de incidencia utilizando los datos de población ocupada de la provincia, así como las razones de incidencia con los intervalos de confianza al 95\% (IC 95\%) para las distintas variables consideradas.

Resultados: Se registraron 645 casos de EEPP, de éstas 607 corresponden a las patologías seleccionadas con una incidencia de 22,67 casos por 100.000 trabajadores. 525 son TME del miembro superior, 68 dermatitis y 14 de asma.

Conclusiones: Los TME del miembro superior representan en la provincia de Alicante la principal EEPP declarada. Se observan diferencias entre los tres grupos de EEPP en Alicante entre 2002-2005, según sexo, edad, actividad económica de la empresa y antigüedad en el puesto de trabajo.

Palabras clave: Enfermedades profesionales, Enfermedades musculoesqueléticas, Dermatitis profesional, Asma, Med Segur Trab 2009; 55 (214): 80-90

Abstract

Objective: Describe the epidemiological pattern of the occupational diseases in the province of Alicante between 2002-2005. 
Methods: Cross-sectional study. The source of information comes from the reports of the occupational diseases registration notified in Alicante 2002-2005. The diseases have been included upper limb musculoskeletal disorders (UL-MSD), dermatitis and asthma. As working and personal factors have been considered age, sex, occupation, firm size and length of employment. The incidence rates were estimated, using workers report of Alicante, and the reasons for incidence with the confidence intervals yours (CI 95\%) for the different variables considered.

Results: 645 cases of occupational diseases were recorded, of which 607 was for the selected pathologies with an incidence of 22.67 cases per 100,000 workers. 525 are of UL-MSD, 68 of Dermatitis and 14 of Asthma.

Conclusions: The UL-MSD represents the main occupational diseases declared in Alicante. There are differences among three groups of occupational diseases between 2002-2005 in Alicante, according to sex, age, economical activity of company and the labour antiquity.

Key words: Occupational Diseases; Musculoskeletal Diseases; Dermatitis, Occupational; Asthma, Med Segur Trab 2009; 55 (214): 80-90 


\section{INTRODUCCIÓN}

El registro de enfermedades profesionales (EEPP) en España constituye el sistema oficial de vigilancia de patologías de origen laboral a nivel nacional. Las estadísticas oficiales incluyen las cifras de las enfermedades notificadas que cumplen los criterios establecidos en el Cuadro de Enfermedades Profesionales (Real Decreto 1995/1978, vigente durante todo el periodo estudiado, y derogado por Real Decreto 1299/2006). Se trata de un registro fiable del que se pueden obtener información de utilidad para explicar la morbilidad laboral ocasionada, tanto por la exposición a determinados riesgos existentes en el lugar de trabajo ${ }^{1}$, como por la existencia de ciertas características individuales que hace que unos trabajadores sean más vulnerables a la aparición de daños en su salud por la práctica de su actividad laboral. La declaración de enfermedad profesional implica un reconocimiento legal que acarrea un trato diferenciado en las prestaciones de la Seguridad Social $^{2}$ con una finalidad indemnizatoria, y que es necesario complementar con acciones preventivas que permitan mejorar las condiciones de trabajo en el puesto en el que se ha declarado.

La incidencia de EEPP en España en el año 2005 fue de 158,28 casos por 100.000 trabajadores ${ }^{3,4}$. Mostrando una distribución muy heterogénea por comunidades autónomas, en el número de casos declarados. Por ejemplo, en Navarra se declararon 753,94 casos por 100.000 trabajadores, mientras que en la Comunidad Valenciana se notificaron 55,96 casos por 100.000 trabajadores. Estas diferencias sugieren que puede existir una infradeclaración ${ }^{5}$ de EEPP en algunas comunidades autónomas, pero también puede producirse como consecuencia de diferencias en la exposición según el patrón económico en las distintas regiones. Sin embargo, la descripción y análisis oficiales de las EEPP no incluyen el análisis completo del parte de declaración, lo que provoca una carencia de conocimiento de las mismas, ya que impide incorporar la perspectiva general de análisis del problema.

Los estudios realizados fuera de España indican que la patología con mayor frecuencia de declaración está formada por los trastornos musculoesqueléticos (TME) que se localizan habitualmente en el miembro superior ${ }^{6}$ afectando especialmente a trabajadores artesanos y de la minería, le sigue en frecuencia la dermatitis profesional ${ }^{7}$ que se origina fundamentalmente en trabajadores de la industria. El asma afecta de forma destacada a panaderos y trabajadores del calzado ${ }^{8}$. Por su parte estudios nacionales han puesto de manifiesto un patrón similar de EEPP declaradas9, 10,11, en el que los TME constituyen la patología más declarada seguida de la dermatitis. En el caso del asma se ha relacionado con una elevada incidencia en los trabajadores de servicios de limpieza y peluquería ${ }^{12}$.

La elaboración y propuesta de políticas laborales y de prevención efectivas, requiere un conocimiento previo de las condiciones de trabajo que influyen en el desarrollo las EEPP, facilitando de esta forma que el diseño y desarrollo de estos programas preventivos, se realicen atendiendo a las necesidades específicas de la población trabajadora.

El objetivo general de este estudio es describir el patrón epidemiológico de las EEPP en la provincia de Alicante entre los años 2002 y 2005. Los objetivos específicos son 1) describir las características de las EEPP declaradas con mayor incidencia en el periodo estudiado, y 2) analizar la relación existente entre determinados factores laborales y personales con la aparición de las EEPP.

\section{MATERIAL Y MÉTODOS}

Se ha realizado un estudio descriptivo transversal de las EEPP declaradas en la provincia de Alicante desde 2002 hasta 2005. La fuente de información la constituyen los partes de enfermedad profesional notificados en el Instituto Valenciano de Seguridad y Salud en el Trabajo (INVASSAT) de Alicante durante el periodo indicado. 
Las variables incluidas en el estudio han sido las siguientes: Demográficas: la edad y el sexo; Variables relacionadas con las condiciones de trabajo, donde se incluye el puesto de trabajo, agrupado según la Clasificación Nacional de Ocupaciones CNO-94 ${ }^{13}$, tamaño de la empresa, antigüedad laboral y actividad económica de la empresa, codificada según la Clasificación Nacional de Actividades Económicas CNAE-93 ${ }^{14}$. Variable de efecto: diagnóstico de la enfermedad profesional, clasificado según la Clasificación Internacional de Enfermedades en su décima revisión (CIE-10).

La inclusión en la base de datos de los códigos CIE-10 se realizó a partir de la revisión efectuada de cada uno partes por un especialista en medicina del trabajo, asignando el código CIE correspondiente en cada caso, ya que en los partes de enfermedad profesional solo figuraba la descripción nominal de la patología y su grupo legal.

A partir de un primer análisis descriptivo de distribución de las EEPP se seleccionaron las más frecuentes, en concreto las que suponen el 94\% de todas las enfermedades registradas durante dicho periodo. Posteriormente se reagruparon en tres grandes grupos de patologías: Trastornos musculoesqueléticos (TME) del miembro superior, incluyéndose en este grupo: el trastorno fibroblástico no especificado (M729), síndrome del manguito rotatorio (M751) tenosinovitis de estiloides radial [de Quervain] (M654), dedo en gatillo (M653), epicondilitis lateral (M771), otras entesopatías, no clasificadas en otra parte (M778), síndrome del túnel carpiano (G560) y lesión del nervio cubital (G562); Dermatitis alérgica de contacto, de causa no especificada (L239); Asma predominantemente alérgica (J450).

A continuación, se realizó un análisis descriptivo para conocer la frecuencia de distribución de las enfermedades según el sexo, edad y tamaño de la empresa. La variable antigüedad en el puesto de trabajo se analizó comparando las medias mediante la $\mathrm{T}$ de student y medianas para los diferentes grupos de enfermedades. También se analizó la distribución de las EEPP agrupadas, en función de la actividad económica de la empresa y de la ocupación del trabajador. Se hicieron pruebas de significación estadística utilizando Chi cuadrado, para el análisis estadístico de los datos se utilizó el programa SPSS 15.0 .

Para estimar las tasas de incidencia se ha utilizado como denominador las cifras absolutas acumuladas de población ocupada de la provincia de Alicante durante los años 2002 al 2005, obtenidos de la Encuesta de la Población Activa ${ }^{4}$, calculando tasas de incidencia por cien mil trabajadores ocupados por sexo, y sectores económicos. Las incidencias por edad y ocupación se calcularon a partir de las cifras absolutas de población ocupada de los años 2002 al 2004 tras su ajuste al periodo analizado. Para las estimaciones de la asociación entre los efectos y las exposiciones se han calculado las razones de tasas y los correspondientes intervalos de confianza al 95\%, el programa usado para el análisis epidemiológico de los datos ha sido Epidat 3.1.

\section{RESULTADOS}

Durante el periodo del estudio se registraron en la provincia de Alicante un total de 645 casos de enfermedad profesional lo que supone una incidencia de 24,09 casos por 100.000 trabajadores.

La distribución de las patologías incluidas en este estudio se presenta en la tabla I. En total, afectaron a 380 hombres $(62,6 \%)$ y 227 mujeres $(37,4 \%)$. El grupo de edad con una frecuencia de presentación de enfermedades más elevado corresponde la población entre 25 y 54 años (485 casos, 79,9\%). Las empresas entre 10 y 49 trabajadores fueron en las que se declararon más casos, concretamente 226 (37,2\%). Respecto a la antigüedad en la empresa en el momento de la declaración de la enfermedad, los trabajadores presentaron una media de 78 meses de antigüedad en la empresa. El $50 \%$ de las enfermedades se produjeron en los 24 primeros meses de actividad laboral. En el grupo de TME del miembro superior 313 casos fueron hombres $(59,6 \%)$ y 212 mujeres $(40,4 \%)$, con una edad de 25 a 54 años en 426 casos $(81,1 \%)$, en las empresas de 10 a 49 trabajadores se 
originaron 193 casos $(36,8 \%)$ seguidas por las de 50 a 249 donde se originaron 148 $(28,2 \%)$, la mediana de la antigüedad cuando se notificó la EEPP fue de 36 meses. El grupo de la dermatitis afectó a 57 hombres $(83,8 \%)$ y 11 mujeres $(16,2 \%)$, en el grupo de edad de 25 a 54 años se produjeron 52 casos (76,5\%), de empresas de 10 a 49 trabajadores procede el mayor número de afectados (27 casos, 39,7\%) y la mediana en el momento de la declaración fue de 16,5 meses. En el grupo de asma por sexo se distribuye en 10 hombres $(71,4 \%)$ y 4 mujeres $(28,6 \%)$, el grupo de edad con mayor declaración fue de 25 a 54 años (7 casos, 50\%) siendo la mediana de 66,5 meses. Se encontraron diferencias estadísticamente significativas por grupos de patologías para las variables sexo $(\mathrm{p}<0,001)$, edad $(\mathrm{p}<0,001)$ y antigüedad en la empresa $(\mathrm{p}<0,01)$.

Tabla I. Distribución de los grupos de enfermedades profesionales declaradas en la provincia Alicante entre 2002 y 2005.

\begin{tabular}{|c|c|c|c|c|c|c|c|c|c|}
\hline \multirow{2}{*}{ Variables } & \multicolumn{2}{|c|}{$\operatorname{Asma}^{1}$} & \multicolumn{2}{|c|}{ Dermatitis $^{2}$} & \multicolumn{2}{|c|}{$\mathbf{T M E}^{3}$} & \multicolumn{2}{|c|}{ Total $^{4}$} & \multirow{2}{*}{$\mathbf{P}$} \\
\hline & $\mathbf{n}$ & $\%$ & $\mathbf{n}$ & $\%$ & $\mathbf{N}$ & $\%$ & $\mathbf{n}$ & $\%$ & \\
\hline \multicolumn{10}{|l|}{ Sexo } \\
\hline Hombres & 10 & 71,4 & 57 & 83,8 & 313 & 59,6 & 380 & 62,6 & \multirow{2}{*}{$<0,001$} \\
\hline Mujeres & 4 & 28,6 & 11 & 16,2 & 212 & 40,4 & 227 & 37,4 & \\
\hline \multicolumn{10}{|l|}{ Edad } \\
\hline 16-19 años & 2 & 14,3 & 3 & 4,4 & 1 & 0,2 & 6 & 1,0 & \multirow{4}{*}{$<0,001$} \\
\hline 20- 24 años & 0 & 0,0 & 7 & 10,3 & 26 & 5,0 & 33 & 5,4 & \\
\hline 25-54 años & 7 & 50,0 & 52 & 76,5 & 426 & 81,1 & 485 & 79,9 & \\
\hline$\geq 55$ años & 5 & 35,7 & 6 & 8,8 & 72 & 13,7 & 83 & 13,7 & \\
\hline \multicolumn{10}{|l|}{ Tamaño de la empresa } \\
\hline$\leq 9$ trabajadores & 5 & 35,7 & 22 & 32,4 & 129 & 24,6 & 156 & 25,7 & \multirow{5}{*}{0,4384} \\
\hline $10-49$ & 6 & 42,9 & 27 & 39,7 & 193 & 36,8 & 226 & 37,2 & \\
\hline $50-249$ & 3 & 21,4 & 17 & 25,0 & 148 & 28,2 & 168 & 27,7 & \\
\hline $250-499$ & 0 & 0,0 & 2 & 2,9 & 38 & 7,2 & 40 & 6,6 & \\
\hline$\geq 500$ trabajadores & 0 & 0,0 & 0 & 0,0 & 17 & 3,2 & 17 & 2,8 & \\
\hline \multicolumn{10}{|c|}{ Antigüedad en la empresa (meses) } \\
\hline $\begin{array}{l}\text { Media (+/-Desviación típica) } \\
\text { Mediana }\end{array}$ & \multicolumn{2}{|c|}{$130,5(125,3)$} & \multicolumn{2}{|c|}{$46,2(83,3)$} & \multicolumn{2}{|c|}{36,0} & \multicolumn{2}{|c|}{36,0} & $<0,01$ \\
\hline \multicolumn{10}{|c|}{$\begin{array}{l}\text { TME = Trastornos musculoesqueléticos del miembro superior: trastorno fibroblástico no especificado (M729), síndrome del } \\
\text { manguito rotatorio (M752), tenosinovitis de estiloides radial [de Quervain] (M654), dedo en gatillo (M653), epicondilitis la- } \\
\text { teral (M771), otras entesopatías no clasificadas en otra parte (M778), síndrome del túnel carpiano (G560) y lesión del nervio } \\
\text { cubital (G562) }\end{array}$} \\
\hline
\end{tabular}

Por lo que respecta a la actividad económica de la empresa, tabla II, se observa que la mayoría de enfermedades se originan en las industrias de productos no metálicos (239 declaraciones, 39,4\% del total), a continuación le siguen las actividades de comercio, reparación de vehículos y hostelería con 114 casos declarados $(18,8 \%)$ y de la construcción con 91 enfermedades notificadas (15\%). Los TME se producen en industrias de productos no metálicos (207 declaraciones, 39,4\%), le siguen las actividades de comercio, reparación de vehículos y hostelería (99 declaraciones, 18,9\%). La dermatitis es declarada en industria de productos no metálicos (23 declaraciones, 33,8\%) seguida de la construcción (21 declaraciones, 30,9\%). El asma se origina mayoritariamente en industrias de productos no 
metálicos (9 declaraciones, 64,3\%). Por grupos de EEPP se observan diferencias estadísticamente significativas $(\mathrm{p}<0,05)$.

Tabla II. Distribución de los grupos de enfermedades profesionales declaradas en la provincia Alicante por actividad económica de la empresa y ocupación del trabajador entre 2002 y 2005.

\begin{tabular}{|c|c|c|c|c|c|c|c|c|c|}
\hline \multirow{2}{*}{ Variables } & \multicolumn{2}{|c|}{$\operatorname{Asma}^{1}$} & \multicolumn{2}{|c|}{ Dermatitis $^{2}$} & \multicolumn{2}{|c|}{$\mathbf{T M E}^{\mathbf{3}}$} & \multicolumn{2}{|c|}{ Total $^{4}$} & \multirow{2}{*}{$\mathbf{P}$} \\
\hline & $\mathbf{n}$ & $\%$ & $\mathbf{N}$ & $\%$ & $\mathbf{n}$ & $\%$ & $\mathbf{n}$ & $\%$ & \\
\hline
\end{tabular}

\section{Actividad económica CNAE-93}

Agricultura, ganadería, pesca, silvicultura y caza

Industrias extractivas

Industrias de productos no metálicos

Industrias de productos metálicos y fabricación muebles

Construcción

Comercio, reparación vehículos, hostelería

Transportes y comunicaciones

Servicios financieros, sociales y administración

Otros servicios

|

\begin{tabular}{|c|c|c|c|}
\hline 0 & 0,0 & 0 & 0,0 \\
\hline 0 & 0,0 & 0 & 0,0 \\
\hline 9 & 64,3 & 23 & 33,8 \\
\hline 1 & 7,1 & 6 & 8,8 \\
\hline 1 & 7,1 & 21 & 30,9 \\
\hline 2 & 14,3 & 13 & 19,1 \\
\hline 0 & 0,0 & 1 & 1,5 \\
\hline 0 & 0,0 & 4 & 5,9 \\
\hline 1 & 7,1 & 0 & 0,0 \\
\hline
\end{tabular}

\begin{tabular}{r|r|}
7 & 1,3 \\
6 & 1,1 \\
207 & 39,4 \\
34 & 6,5 \\
69 & 13,1 \\
99 & 18,9 \\
11 & 2,1 \\
71 & 13,5 \\
21 & 4,0
\end{tabular}

\begin{tabular}{r|r|}
7 & 1,2 \\
6 & 1,0 \\
239 & 39,4 \\
41 & 6,8 \\
91 & 15,0 \\
114 & 18,8 \\
12 & 2,0 \\
75 & 12,4 \\
22 & 3,6
\end{tabular}

Ocupación CNO-94

Dirección de las empresas y de la Administración Pública

Técnicos y profesionales científicos e intelectuales

Técnicos y profesionales de apoyo

Empleados de tipo administrativo

Trabajadores de servicios de restauración, personales, protección y vendedores de comer.

Trabajadores cualificados en agricultura y pesca

Artesanos y trabajadores cualificados de industrias manufactureras, construcción y minería, exp. operadores

Operadores de instalaciones y maquinaria; montadores

Trabajadores no cualificados

\begin{tabular}{|c|c|c|c|c|c|c|c|c|}
\hline 0 & 0,0 & 0 & 0,0 & 2 & 0,4 & 2 & 0,3 & \\
\hline 0 & 0,0 & 0 & 0,0 & 1 & 0,2 & 1 & 0,2 & \\
\hline 1 & 7,1 & 0 & 0,0 & 6 & 1,1 & 7 & 1,2 & \\
\hline 0 & 0,0 & 0 & 0,0 & 11 & 2,1 & 11 & 1,8 & \\
\hline 2 & 14,3 & 7 & 10,3 & 61 & 11,6 & 70 & 11,5 & 0,1009 \\
\hline 0 & 0,0 & 0 & 0,0 & 3 & 0,6 & 3 & 0,5 & \\
\hline 9 & 64,3 & 41 & 60,3 & 202 & 38,5 & 252 & 41,5 & \\
\hline 2 & 14,3 & 12 & 17,6 & 151 & 28,8 & 165 & 27,2 & \\
\hline 0 & 0,0 & 8 & 11,8 & 88 & 16,7 & 96 & 15,8 & \\
\hline
\end{tabular}

Asma = asma predominantemente alérgica (J450)

Dermatitis = dermatitis alérgica de contacto, de causa no especificada (L239);

TME = Trastornos musculoesqueléticos del miembro superior: trastorno fibroblástico no especificado (M729), síndrome del manguito rotatorio (M752), tenosinovitis de estiloides radial [de Quervain] (M654), dedo en gatillo (M653), epicondilitis lateral (M771), otras entesopatías no clasificadas en otra parte (M778), síndrome del túnel carpiano (G560) y lesión del nervio cubital (G562)

4 El total de los tres grupos de enfermedades incluye al el $94 \%$ de todas las EEPP declaradas.

Atendiendo a la ocupación de la población trabajadora, se observa que en los tres grupos de enfermedades, la declaración se produce fundamentalmente en dos grupos de ocupaciones: artesanos y trabajadores cualificados de la industria manufacturera, construcción y minería (252 casos, 41,5\%), seguido del grupo de operadores de 
instalaciones maquinaria y montadores (165 casos, 27,2\%). Para los tres grupos de EEPP la ocupación que más casos genera es la de artesanos y trabajadores cualificados de la industria manufacturera, construcción y minería (202 casos, 38,5\% en TME; 41 casos, $60,3 \%$ en dermatitis y 9 declaraciones, 64,3\% para el asma). La tabla III, presenta la distribución de patologías incluidas en el grupo de los TME del miembro superior. Se observa que las patologías declaradas dentro de este grupo corresponden en un $69,71 \%$ con enfermedades de tipo osteomuscular, fundamentalmente síndrome del manguito rotatorio $(47,6 \%)$ y epicondilitis lateral $(17,5 \%)$, y en un $30,29 \%$ por enfermedades de tipo neurológico, mayoritariamente síndrome del túnel carpiano $(29,7 \%)$.

Tabla III. Distribución de los diagnósticos incluidos en el grupo de trastornos musculoesqueléticos del miembro superior.

\begin{tabular}{lcc}
\hline \multicolumn{1}{c}{ TME del miembro superior } & n & \% \\
\hline Síndrome del manguito rotatorio (M752) & 250 & 47,6 \\
Síndrome del túnel carpiano (G560) & 156 & 29,7 \\
Epicondilitis lateral (M771) & 92 & 17,5 \\
Tenosinovitis de estiloides radial [de Quervain] (M654) & 10 & 1,9 \\
Trastorno fibroblástico no especificado (M729) & 9 & 1,7 \\
Otras entesopatías, no clasificadas en otra parte (M778) & 3 & 0,6 \\
Lesión del nervio cubital (G562) & 3 & 0,6 \\
Dedo en gatillo (M653) & 2 & 0,4 \\
Total & $\mathbf{5 2 5}$ & $\mathbf{1 0 0}$ \\
\hline
\end{tabular}

Tabla IV. Tasas de incidencia de los grupos de enfermedades profesionales por 100.000 trabajadores en la provincia Alicante entre 2002 y 2005.

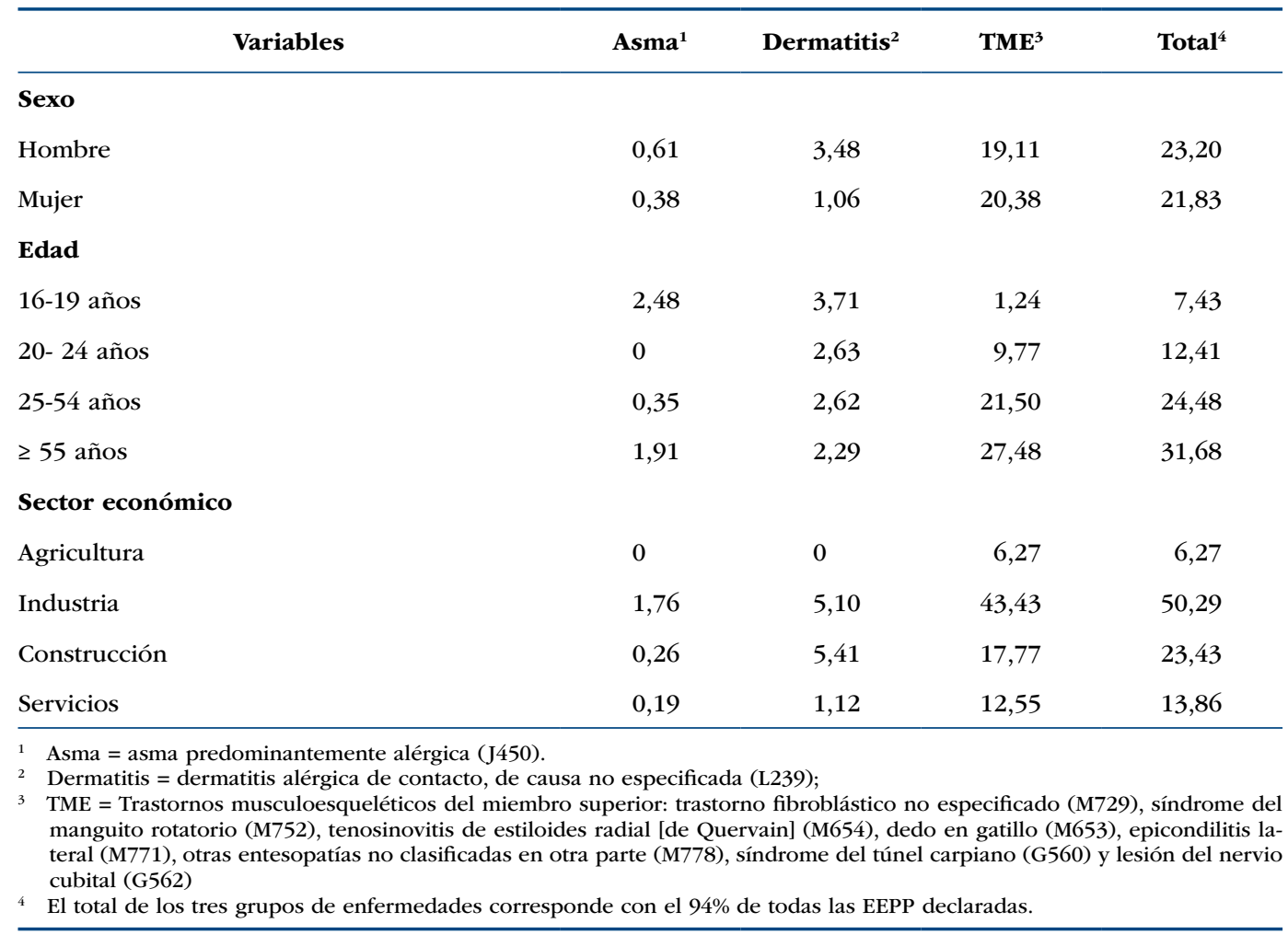


La incidencia más elevada (tabla IV) la presentan los TME, siendo mas alta en mujeres 20,38 EEPP por 100.000 trabajadoras respecto de los hombres $(19,11)$. Para el asma y la dermatitis son superiores las cifras en hombres. En el caso de la dermatitis la incidencia en hombres $(3,48)$ triplica a la de las mujeres $(1,06)$.

Al comparar la incidencia de patologías por grupos de edad se observa que en el caso de los TME las incidencias más elevadas se producen en los trabajadores mayores de 55 años (27,48 EEPP por 100.000 trabajadores), mientras que en el caso de la dermatitis y el asma se originan con mayor frecuencia entre los jóvenes. El sector de la industria presenta las tasas más altas para los tres grupos de enfermedades, en el caso de la dermatitis también son elevadas las tasas en el sector de la construcción.

En relación a la ocupación del trabajador (tabla V), se observa que la mayor incidencia de EEPP se produce en artesanos y trabajadores cualificados de industrias manufactureras, construcción y minería, excepto operadores; en el caso del asma $(1,96)$ y de la dermatitis $(8,94)$, mientras que para los TME son los operadores de instalaciones y maquinaria los que tienen mayor incidencia $(45,70)$.

Los hombres presentaran un riesgo significativamente mayor de dermatitis (tabla VI) (RT 3,29; IC 95\% 1,73-6,27). Con respecto a la categoría de referencia, 25-54 años, los trabajadores de mas de 55 años presentan más riesgo de asma y TME, mientras que los más jóvenes (menores de 25 años) presentan menor riesgo en el caso de los TME. Tomando como referencia a los trabajadores del sector servicios, se observa que los trabajadores de la industria muestran mayor riesgo de TME del miembro superior (RT 3,46; IC 95\% 2,874,17) y asma (RT 9,43; IC 95\% 2,43-53,34), el sector de la construcción también muestra un incremento del riesgo para la dermatitis en relación al sector servicios, y es el que obtiene una razón de tasas más elevada (4,83; IC 95\% 2,45-9,62).

Tabla V. Tasas de incidencia de los grupos de enfermedades profesionales por 100.000 trabajadores por ocupación del trabajador en la provincia Alicante entre 2002 y 2005.

\begin{tabular}{|c|c|c|c|c|}
\hline Variable & Asma $^{1}$ & Dermatitis $^{2}$ & TME $^{3}$ & Total $^{4}$ \\
\hline \multicolumn{5}{|l|}{ Ocupación } \\
\hline $\begin{array}{l}\text { Dirección de las empresas y de la Administra- } \\
\text { ción Pública }\end{array}$ & 0 & 0 & 0,89 & 0,89 \\
\hline $\begin{array}{l}\text { Técnicos y profesionales científicos e intelec- } \\
\text { tuales }\end{array}$ & 0 & 0 & 0,40 & 0,40 \\
\hline Técnicos y profesionales de apoyo & 0,61 & 0 & 3,68 & 4,30 \\
\hline Empleados de tipo administrativo & 0 & 0 & 4,27 & 4,27 \\
\hline $\begin{array}{l}\text { Trabajadores de servicios de restauración, per- } \\
\text { sonales, protección y vendedores de comer. }\end{array}$ & 0,52 & 1,82 & 15,86 & 18,20 \\
\hline $\begin{array}{l}\text { Trabajadores cualificados en agricultura y } \\
\text { pesca }\end{array}$ & 0 & 0 & 5,73 & 5,73 \\
\hline $\begin{array}{l}\text { Artesanos y trabajadores cualificados de } \\
\text { industrias manufactureras, construcción y } \\
\text { minería, exp. operadores }\end{array}$ & 1,96 & 8,94 & 44,05 & 54,96 \\
\hline $\begin{array}{l}\text { Operadores de instalaciones y maquinaria; } \\
\text { montadores }\end{array}$ & 0,61 & 3,63 & 45,70 & 49,94 \\
\hline Trabajadores no cualificados & 0 & 1,71 & 18,76 & 20,46 \\
\hline \multicolumn{5}{|c|}{$\begin{array}{l}\text { TME = Trastornos musculoesqueléticos del miembro superior: trastorno fibroblástico no especificado (M729), síndrome del } \\
\text { manguito rotatorio (M752), tenosinovitis de estiloides radial [de Quervain] (M654), dedo en gatillo (M653), epicondilitis la- } \\
\text { teral (M771), otras entesopatías no clasificadas en otra parte (M778), síndrome del túnel carpiano (G560) y lesión del nervio } \\
\text { cubital (G562) }\end{array}$} \\
\hline
\end{tabular}


Tabla VI. Razones de incidencia e intervalos de confianza al $95 \%$ en los grupos de enfermedades profesionales declaradas en la provincia Alicante entre 2002 y 2005.

\begin{tabular}{|c|c|c|c|}
\hline Variables & $\begin{array}{c}\left.\text { RT (IC }{ }_{95 \%}\right) \\
\text { Asma }^{1}\end{array}$ & $\begin{array}{c}\left.\text { RT (IC }{ }_{95 \%}\right) \\
\text { Dermatitis }^{2}\end{array}$ & $\begin{array}{c}\text { RT }\left(\text { IC }_{95 \%}\right) \\
\text { TME }^{3}\end{array}$ \\
\hline \multicolumn{4}{|l|}{ Sexo } \\
\hline Mujer & 1,00 & 1,00 & 1,00 \\
\hline Hombre & $1,59(0,50-5,06)$ & $3,29(1,73-6,27)$ & $0,94(0,79-1,11)$ \\
\hline \multicolumn{4}{|l|}{ Edad } \\
\hline 16-19 años & $7,01(0,71-36,79)$ & $1,41(0,28-4,37)$ & $0,06(0,01-0,41)$ \\
\hline 20- 24 años & - & $1,00(0,46-2,21)$ & $0,45(0,31-0,68)$ \\
\hline 25-54 años & 1,00 & 1,00 & 1,00 \\
\hline$\geq 55$ años & $5,40(1,35-19,77)$ & $0,87(0,37-2,03)$ & $1,28(1,00-1,64)$ \\
\hline \multicolumn{4}{|l|}{ Sector económico } \\
\hline Agricultura & - & - & $0,50(0,24-1,06)$ \\
\hline Industria & $9,43(2,43-53,34)$ & $4,56(2,53-8,21)$ & $3,46(2,87-4,17)$ \\
\hline Construcción & $1,38(0,03-17,20)$ & $4,83(2,45-9,62)$ & $1,41(1,08-1,86)$ \\
\hline Servicios & 1,00 & 1,00 & 1,00 \\
\hline $\begin{array}{ll}1 & \text { Asma = asma predominant } \\
2 & \text { Dermatitis = dermatitis alé } \\
3 & \text { TME = Trastornos musculo } \\
\text { manguito rotatorio (M752) } \\
\text { teral (M771), otras entesop } \\
\text { cubital (G562) } \\
4 & \text { El total de los tres grupos } \\
5 & \text { RT= razón de tasas. IC 95\% }\end{array}$ & $\begin{array}{l}J 450) \text {. } \\
\text { de causa no especific } \\
\text { niembro superior: tras } \\
\text { estiloides radial [de Q } \\
\text { as en otra parte ( } M 778 \\
\text { corresponde con el } 94 \\
\text { nfianza al } 95 \% \text {. }\end{array}$ & $\begin{array}{l}\text { 239); } \\
\text { fibroblástico no espe } \\
\text { in] (M654), dedo en } \\
\text { lrome del túnel carp } \\
\text { todas las EEPP decla }\end{array}$ & $\begin{array}{l}\text { do (M729), síndrome del } \\
\text { (M653), epicondilitis la- } \\
\text { 560) y lesión del nervio }\end{array}$ \\
\hline
\end{tabular}

\section{DISCUSIÓN}

Los TME del miembro superior representan en la provincia de Alicante el principal grupo de patología laboral declarada. Los sectores con mas riesgo son la construcción e industria.

El hecho de que más del $60 \%$ de las patologías notificadas se originen en pequeñas y medianas empresas de menos de 50 trabajadores, podría deberse a que los trabajadores de estas empresas reciben una menor formación e información en materia preventiva que los pertenecientes a grandes empresas como se observa en los resultados de la VI Encuesta Nacional de Condiciones de Trabajo (ENCT) ${ }^{15}$.

Cuando se comparan los datos obtenidos con otros estudios existe concordancia para las ocupaciones que tienen mayor número de declaraciones, así como en el tipo de patología declarada mayoritariamente, enfermedades leves que provocan daño de tipo osteomuscular, seguidas de las de la piel y neuropatías periféricas $(1,7,10,11)$. También a nivel nacional se observan resultados muy similares en cuanto a la distribución por sexo, al observar mayor incidencia de TME en mujeres, siendo superiores en hombres para el asma y la dermatitis ${ }^{16}$. Estas diferencias por sexo podrían ser explicadas por factores laborales relacionados con una segregación laboral por género, originándose de esta forma diferentes patrones de morbilidad laboral en hombres y en mujeres. Para profundizar en el conocimiento del origen de esa diferenciación será necesario realizar más estudios específicos que analicen las patologías de origen laboral desde una perspectiva de género.

Respecto a la distribución de las patologías del miembro superior es frecuente que se localicen en el hombro, situándose el síndrome del manguito rotatorio como la 
patología laboral más declarada como EEPP en la provincia de Alicante en el periodo 2002-2005. En una revisión realizada por Grooten ${ }^{17}$ se encontraron evidencias de que un elevado esfuerzo físico, la realización de trabajos repetitivos y adoptar posturas de cuello inadecuadas se consideran factores de riesgo para sufrir dolor de hombro, a lo que se une la elevada carga mental como otro factor de riesgo que frecuentemente se combina con los clásicos riesgos físicos, incrementando notablemente los efectos de dicha exposición. Los resultados de nuestro estudio son compatibles con esa afirmación, al observarse una mayor incidencia de TME de miembro superior entre los trabajadores que ocupan puestos de trabajo de inferior cualificación y ser estos trabajadores los que habitualmente se encuentran más expuestos a los riesgos físicos indicados por Grooten.

La introducción de los códigos CIE 10 en la base de datos utilizada ha permitido que se pudiera diferenciar la localización de patologías incluidas en un mismo grupo, diferenciación que no era posible realizar con el anterior cuadro de EEPP, de esta forma se ha podido visualizar la importancia relativa que tiene de la patología de hombro entre las EEPP registradas, y a la que por su destacable peso específico dentro del conjunto de TME (250 casos, 47,6\% del grupo TME del miembro superior) se le debería dedicar un especial seguimiento por medio de estudios específicos.

Son destacables las diferencias encontradas en las tasas de incidencias de EEPP por ocupaciones para trabajadores de construcción y minería (54,96 EEPP por 100.000 trabajadores), administrativos $(4,27)$ y dirección de empresas $(0,89)$, respecto a los datos reflejados en la VI ENCT donde los trabajadores de la construcción y minería (3,3\%), administrativos (3\%) y directivos de empresas $(2,8 \%)$ responden en proporciones muy similares al ser preguntados si habían sido diagnosticados o estaban en trámite para reconocerle una enfermedad profesional ${ }^{15}$. Sin embargo, los resultados deben interpretarse con cautela, principalmente debido a las posibles diferencias en los factores de comparación. Esto podría profundizarse a través de estudios posteriores que incluyan seguimientos del trabajador según ocupación y sus condiciones específicas de trabajo y su relación con la frecuencia y aparición de EEPP.

Dentro de las limitaciones del estudio están el no disponer de información del grado de exposición real al que han estado expuestos los trabajadores. La utilización del parte de declaración no ha permitido disponer de otras variables individuales como nacionalidad o discapacidad, o de variables laborales como modalidad de contrato, tipo de jornada laboral, subcontratación, o de trabajos realizados con anterioridad; que podrían estar condicionando la probabilidad de desarrollar una enfermedad profesional ${ }^{10}$. Además a través de la información contenida en el parte de declaración utilizado tenemos la limitación de no poder estimar ni la gravedad del proceso, mediante la duración de la baja ni el resultado final que se derive del mismo, ya sea con una incapacidad parcial, incapacidad permanente, o reincorporación al trabajo sin restricciones.

La utilización de la Encuesta de Población Activa para los datos de población ocupada ofrece una adecuada validez a nivel provincial, sin embargo tiene el inconveniente de ofrecer escasos datos provinciales desagregados ${ }^{4}$. Por otra parte los datos de población ocupada de la EPA resultan indicados para enfermedades con corto periodo de latencia, pero no resultan adecuados para patologías con largos periodos de latencia como en el caso del asma ${ }^{11}$, por los que las tasas calculadas para esta patología deberían ser analizadas con precaución.

Parece necesario mejorar el enfoque medico preventivo de la enfermedad profesional, creando sistemas de información que profundicen en los factores de riesgo de las patologías declaradas y sobre sus características médico laborales, para permitir la posterior aplicación de programas específicos de prevención que consigan reducir la incidencia y gravedad de las EEPP.

Dentro del panorama económico de la provincia de Alicante, además de la notable importancia de los servicios donde destaca el sector turístico, tienen especial relevancia para el comercio exterior dos sectores industriales: el del calzado y el de la piedra natural, ya que éstos ocupan las primeras posiciones en lo que respecta a exportaciones industriales 
de productos alicantinos ${ }^{18}$. Son precisamente en estos dos sectores donde se generaron más del 20\% de casos de EEPP en los grupos seleccionados durante el periodo 2002 a 2005, por lo que las intervenciones preventivas deberían priorizarse sobre los mismos y tendrían que ser valoradas como acciones de ayuda para mejorar la competitividad de estos sectores ante situaciones de desaceleración económica.

\section{REFERENCIAS}

1. Roel-Valdés J, Arizo-Luque V, Ronda-Pérez E. Epidemiología del síndrome del túnel carpiano de origen laboral en la provincia de Alicante, 1996-2004. Rev Esp Salud Pública. 2006; 80: 395-409.

2. Real Decreto Legislativo 1/1994, de 20 de junio, por el que se aprueba el Texto Refundido de la Ley General de la Seguridad Social. BOE No. 154, (29 Jun 1994).

3. Anuario de estadísticas laborales y de asuntos sociales 2005. Ministerio de Trabajo e Inmigración. [citado 20 Jun 2008]. Disponible en: http://www.mtin.es/estadisticas/ anuario2005 /ATE/index.htm.

4. Encuesta de Población Activa. Instituto Nacional de Estadística. [citado 20 Jun 2008]. Disponible en: http://www.ine.es.

5. García AM, Gadea R. Estimación de la mortalidad y morbilidad por enfermedades laborales en España. Arch Prev Riesgos Labor. 2004; 7: 3-8.

6. Cherry NM, Meyer JD, Chen Y, Holt DL, McDonald JC. The reported incidence of work-related musculoskeletal disease in the UK: MOSS 1997-2000. Occup Med. 2001; $51: 450-5$

7. Cherry NM, Meyer JD, Holt DL, Chen Y, McDonald JC. Surveillance of work-related diseases by occupational physicians in the UK: OPRA 1996-1999. Occup Med. 2000; 50 :496-503.

8. Karjalainen A, Kurppa K, Martikainen R, Karjalainen J, Klaukka T. Exploration of asthma risk by occupationextended analysis of an incidence study of the Finnish population. Scand J Work Environ Health. 2002; 28 : $49-57$.

9. Ordaz E, Maqueda J. Análisis de la incidencia y tendencia de las enfermedades profesionales en España: 1998-2003. Med Segur Trab 2005; 199: 41-51.

10. Gil G, García ML, López R. Análisis de las enfermedades profesionales declaradas en los trabajadores inmigrantes de la Región de Murcia durante el periodo 2000-2003. Med Segur Trab. 2005; 51: 31-42.

11. García M, Castañeda R. Enfermedades profesionales declaradas en hombres y mujeres en España en 2004. Rev Esp Salud Pública. 2006; 80: 361-75.

12. Orriols R, Costa R, Albanell M, Alberti C, Castejón J, Monso E, Panades R, Rubira N, Zock JP; Malaltia Ocupacional Respiratoria (MOR) Group. Reported occupational respiratory diseases in Catalonia. Occup Environ Med. 2006; 63: 255-60.

13. Real Decreto 917/1994, de 6 de mayo, por el que se aprueba la Clasificación Nacional de Ocupaciones 1994 (CNO-94). BOE No. 126, (27 May 1994).

14. Real Decreto 1560/1992, de 18 de diciembre, por el que se aprueba la Clasificación Nacional de Actividades Económicas (CNAE-93), BOE No. 306, (22 Dic1992).

15. VI Encuesta Nacional de Condiciones de Trabajo. Instituto Nacional de Seguridad e Higiene en el Trabajo; Madrid. 2007. [citado 20 Jun 2008]. Disponible en http://www.mtas.es/insht/statistics/viencuesta.pdf.

16. Zimmermann M, González MF, Martínez V. Las condiciones de trabajo según la perspectiva de género: las implicaciones y repercusiones sobre la morbilidad. La Mutua. 2006; 14: 91-103.

17. Grooten W. Work and neck/shoulder pain risk and prognostic factor. Karolinska institutet. Stockholm; 2006.

18. Gabinete de estudios. Alicante en cifras 2006. Cámara Oficial de Comercio Industria y Navegación de Alicante. [citado 27 Jul 2008]. Disponible en http://www.camaralicante.com/IE/do?paginaref=AENC\&IDDoc umento $=2246$. 